\title{
A SINGULAR CONTROL PROBLEM WITH AN EXPECTED AND A PATHWISE ERGODIC PERFORMANCE CRITERION
}

ANDREW JACK AND MIHAIL ZERVOS

Received 13 May 2005; Revised 12 April 2006; Accepted 13 April 2006

We consider the problem of controlling a general one-dimensional Itô diffusion by means of a finite-variation process. The objective is to minimise a long-term average expected criterion as well as a long-term pathwise criterion that penalise deviations of the underlying state process from a given nominal point as well as the expenditure of control effort. We solve the resulting singular stochastic control problems under general assumptions by identifying an optimal strategy that is explicitly characterised.

Copyright (C) 2006 A. Jack and M. Zervos. This is an open access article distributed under the Creative Commons Attribution License, which permits unrestricted use, distribution, and reproduction in any medium, provided the original work is properly cited.

\section{Introduction}

We consider a stochastic system, the state of which is modelled by the controlled, onedimensional Itô diffusion

$$
d X_{t}=b\left(X_{t}\right) d t+d \xi_{t}+\sigma\left(X_{t}\right) d W_{t}, \quad X_{0}=x \in \mathbb{R},
$$

where $W$ is a standard, one-dimensional Brownian motion, and the controlled process $\xi$ is a càglàd finite-variation process. The objective of the optimisation problem is to minimise the long-term average expected performance criterion

$$
\limsup _{T \rightarrow \infty} \frac{1}{T} E\left[\int_{0}^{T} h\left(X_{s}\right) d s+\int_{[0, T]} k_{+}\left(X_{s}\right) \oplus d \xi_{s}^{+}+\int_{[0, T]} k_{-}\left(X_{s}\right) \ominus d \xi_{s}^{-}\right]
$$

as well as the long-term average pathwise performance criterion

$$
\limsup _{T \rightarrow \infty} \frac{1}{T}\left[\int_{0}^{T} h\left(X_{s}\right) d s+\int_{[0, T]} k_{+}\left(X_{s}\right) \oplus d \xi_{s}^{+}+\int_{[0, T]} k_{-}\left(X_{s}\right) \ominus d \xi_{s}^{-}\right]
$$


over all admissible choices of $\xi$. Here, $h$ is a given positive function that is strictly decreasing in ] $-\infty, 0$ [ and strictly increasing in ] $0, \infty$ [. Thus, these performance indices penalise deviations of the state process $X$ from the nominal operating point 0 . Given a finite-variation process $\xi$, we denote by $\xi^{+}$and $\xi^{-}$the unique processes that provide the minimal decomposition of $\xi$ into the difference of two increasing processes, and

$$
\begin{aligned}
& \int_{[0, T]} k_{+}\left(X_{s}\right) \oplus d \xi_{s}^{+}:=\int_{0}^{T} k_{+}\left(X_{s}\right) d\left(\xi^{+}\right)_{s}^{c}+\sum_{0 \leq s \leq T} \int_{0}^{\Delta\left(\xi^{+}\right)_{s}} k_{+}\left(X_{s}+r\right) d r, \\
& \int_{[0, T]} k_{-}\left(X_{s}\right) \ominus d \xi_{s}^{-}:=\int_{0}^{T} k_{-}\left(X_{s}\right) d\left(\xi^{-}\right)_{s}^{c}+\sum_{0 \leq s \leq T} \int_{0}^{\Delta\left(\xi^{-}\right)_{s}} k_{-}\left(X_{s}-r\right) d r,
\end{aligned}
$$

where $\left(\xi^{ \pm}\right)^{c}$ are the continuous parts of $\xi^{ \pm}$. The functions $k_{+}$and $k_{-}$represent the proportional costs associated with the use of the control process $\xi$ to push the left-continuous state process $X$ in the positive and negative directions, respectively.

Singular stochastic control with an expected discounted criterion was introduced by Bather and Chernoff [1] who considered a simplified model of spaceship control. In their seminal paper, Beneš et al. [2] were the first to solve rigorously an example of a finite-fuel singular control problem. Since then, the area has attracted considerable interest in the literature. Karatzas [9], Harrison and Taksar [8], Shreve et al. [17], Chow et al. [3], Sun [19], Soner and Shreve [18], Ma [11], Zhu [21], and Fleming and Soner [7, Chapter VIII] provide an incomplete list, in chronological order, of further important contributions.

The first singular control problem with an ergodic expected criterion was solved by Karatzas [9], who considered the control of a standard Brownian motion. Menaldi and Robin [12] later established the existence of an optimal control to the problem that we consider when $b$ and $\sigma$ are Lipshitz continuous with $\sigma$ bounded, $k_{+} \equiv k_{-} \equiv 0$, and under other technical conditions. Also, Weerasinghe [20] solved the version of the problem that arises when the drift is controllable in a bang-bang sense and the functions $k_{+}$and $k_{-}$are both equal to the same constant $K$.

At this point, we should note that, to the best of our knowledge, our analysis provides the first model of singular control with an ergodic pathwise criterion to be considered in the literature. Other stochastic control problems with an ergodic pathwise criterion have recently attracted significant interest. Notable contributions in this area include Rotar [15], Presman et al. [13], Dai Pra et al. [4], Dai Pra et al. [5], and a number of references therein.

We solve the problems that we consider and we derive an explicit characterisation of an optimal strategy by finding an appropriate solution to the associated HamiltonJacobi-Bellman (HJB) equation under general assumptions. When the cost functions $k_{+}$ and $k_{-}$are both equal to a constant $K$, our assumptions regarding the rest of the problem data are similar to those imposed by Weerasinghe [20]. However, we should note that, in this special case, the problem with the expected performance criterion that we solve is fundamentally different from the one solved by Weerasinghe [20], which is evidenced by the fact that the two problems are associated with different HJB equations.

With regard to the structure of the performance criteria that we consider, penalising the expenditure of control effort by means of integrals as in (1.4) was introduced by 
Zhu [21] and was later adopted by Davis and Zervos [6]. An apparently more natural choice for penalising control effort expenditure would arise if we replaced these integrals by

$$
\int_{[0, T]} k_{+}\left(X_{s}\right) d \xi_{s}^{+}, \quad \int_{[0, T]} k_{-}\left(X_{s}\right) d \xi_{s}^{-},
$$

respectively. However, such a choice would lead to a less "pleasant" HJB equation that we cannot solve. Also, it is worth noting that the two types of integrals are identical when the functions $k_{+}$and $k_{-}$are both constant. At this point, we should note that our assumptions allow for the possibility that the uncontrolled diffusion associated with (1.1) explodes in finite time. In such a case, it turns out that an optimal control is a stabilising one.

\section{The singular stochastic control problem}

We consider a stochastic system, the state process $X$ of which is driven by a Brownian motion $W$ and a controlled process $\xi$. In particular, we consider the controlled, onedimensional SDE

$$
d X_{t}=b\left(X_{t}\right) d t+d \xi_{t}+\sigma\left(X_{t}\right) d W_{t}, \quad X_{0}=x \in \mathbb{R},
$$

where $b, \sigma: \mathbb{R} \rightarrow \mathbb{R}$ are given functions, and $W$ is a standard, one-dimensional Brownian motion. Here, the singular control process $\xi$ is a càglàd finite-variation process, the time evolution of which is determined by the system's controller. Given such a process, we denote by $\xi=\xi^{+}-\xi^{-}$the unique decomposition of $\xi$ into the difference of two increasing processes $\xi^{+}$and $\xi^{-}$such that the total variation process $\check{\xi}$ of $\xi$ is given by $\check{\xi}=\xi^{+}+\xi^{-}$.

We adopt a weak formulation of the control problem that we study.

Definition 2.1. Given an initial condition $x \in \mathbb{R}$ a control of a stochastic system governed by dynamics as in $(2.1)$ is an octuple $\mathbb{C}_{x}=\left(\Omega, \mathscr{F}_{,}, \mathscr{F}_{t}, P, W, \xi, X, \tau\right)$, where $\left(\Omega, \mathscr{F}, \mathscr{F}_{t}, P\right)$ is a filtered probability space satisfying the usual conditions, $W$ is a standard one-dimensional $\left(\mathscr{F}_{t}\right)$-Brownian motion, $\xi$ is a finite-variation $\left(\mathscr{F}_{t}\right)$-adapted càglàd process, and $X$ is an $\left(\mathscr{F}_{t}\right)$-adapted càglàd process that satisfies $(2.1)$ up to its possible explosion time $\tau$.

Define $\mathscr{C}_{x}$ to be the family of all such controls $\mathbb{C}_{x}$.

With each control $\mathbb{C}_{x} \in \mathscr{C}_{x}$, we associate the long-term average expected performance criterion $J^{\mathrm{E}}\left(\mathbb{C}_{x}\right)$ defined by

$$
J^{\mathrm{E}}\left(\mathbb{C}_{x}\right)=\limsup _{T \rightarrow \infty} \frac{1}{T} E\left[\int_{0}^{T} h\left(X_{s}\right) d s+\int_{[0, T]} k_{+}\left(X_{s}\right) \oplus d \xi_{s}^{+}+\int_{[0, T]} k_{-}\left(X_{s}\right) \ominus d \xi_{s}^{-}\right]
$$

if $P(\tau=\infty)=1$, and by

$$
J^{\mathrm{E}}\left(\mathbb{C}_{x}\right)=\infty, \quad \text { if } P(\tau=\infty)<1,
$$


as well as the long-term average pathwise criterion

$$
J^{\mathrm{P}}\left(\mathbb{C}_{x}\right)=\infty \mathbf{1}_{\{\tau<\infty\}}+\limsup _{T \rightarrow \infty} \frac{1}{T}\left[\int_{0}^{T} h\left(X_{s}\right) d s+\int_{[0, T]} k_{+}\left(X_{s}\right) \oplus d \xi_{s}^{+}+\int_{[0, T]} k_{-}\left(X_{s}\right) \ominus d \xi_{s}^{-}\right],
$$

which is a random variable with values in $[0, \infty]$. Here, $h: \mathbb{R} \rightarrow \mathbb{R}$ is a given function that models the running cost resulting from the system's operation, while $k_{+}, k_{-}$are given functions penalising the expenditure of control effort. The integrals with respect to $\xi^{+}$ and $\xi^{-}$are defined by (1.4) in the introduction, respectively.

The objective is to minimise the performance criteria defined by (2.2)-(2.3) and (2.4) over all admissible controls $\mathbb{C}_{x} \in \mathscr{C}_{x}$. We impose the following assumption on the problem data.

Assumption 2.2. The following conditions hold.

(a) The functions $b, \sigma: \mathbb{R} \rightarrow \mathbb{R}$ are continuous, and there exists a constant $C_{1}>0$ such that

$$
0<\sigma^{2}(x) \leq C_{1}(1+|x|), \quad \forall x \in \mathbb{R}
$$

(b) The function $h$ is continuous, strictly decreasing on ] $-\infty, 0$ [ and strictly increasing on $] 0, \infty\left[\right.$. Also, $h(0)=0$ and there exists a constant $C_{2}>0$ such that

$$
h(x) \geq C_{2}(|x|-1), \quad \forall x \in \mathbb{R} .
$$

(c) Given any constant $\gamma>0$,

$$
\lim _{x \rightarrow \pm \infty} \frac{1}{\sigma^{2}(x)}[h(x)-\gamma|b(x)|]=\infty .
$$

(d) The functions $k_{+}$and $k_{-}$are $C^{1}$ and there exists a constant $C_{3}>0$ such that

$$
\left.\left.k_{+}(x), k_{-}(x) \in\right] 0, C_{3}\right], \quad \forall x \in \mathbb{R} .
$$

(e) There exist $\alpha_{-} \leq \alpha_{+}$such that the function

$$
-\frac{1}{2} \sigma^{2} k_{+}^{\prime}-b k_{+}+h\left\{\begin{array}{l}
\text { is strictly decreasing and positive in }]-\infty, \alpha_{-}[, \\
\text {is strictly negative inside }] \alpha_{-}, \alpha_{+}\left[, \text {if } \alpha_{-}<\alpha_{+},\right. \\
\text {is strictly increasing and positive in }] \alpha_{+}, \infty[.
\end{array}\right.
$$

(f) There exist $a_{-} \leq a_{+}$such that the function

$$
\frac{1}{2} \sigma^{2} k_{-}^{\prime}+b k_{-}+h\left\{\begin{array}{l}
\text { is strictly decreasing and positive in }]-\infty, a_{-}[, \\
\text {is strictly negative inside }] a_{-}, a_{+}\left[\text {, if } a_{-}<a_{+},\right. \\
\text {is strictly increasing and positive in }] a_{+}, \infty[.
\end{array}\right.
$$


Note that Assumption 2.2(a) implies the nondegeneracy condition (ND)' and the local integrability condition (LI) ${ }^{\prime}$ in [10, Section 5.5 of Karatzas and Shreve]. It follows that, given an initial condition $x \in \mathbb{R}$, the uncontrolled diffusion

$$
d X_{t}=b\left(X_{t}\right) d t+\sigma\left(X_{t}\right) d W_{t}, \quad X_{0}=x,
$$

has a unique weak solution up to a possible explosion time. Moreover, the scale function and the speed measure that characterise a one-dimensional diffusion such as the one in (2.11), which are given by

$$
\begin{gathered}
p_{x_{0}}\left(x_{0}\right)=0, p_{x_{0}}^{\prime}(x)=\exp \left(-2 \int_{x_{0}}^{x} \frac{b(s)}{\sigma^{2}(s)} d s\right), \quad \text { for } x \in \mathbb{R}, \\
m_{x_{0}}(d x)=\frac{2}{p_{x_{0}}^{\prime}(x) \sigma^{2}(x)} d x,
\end{gathered}
$$

respectively, for any given choice of $x_{0} \in \mathbb{R}$, are well defined.

At this point it is worth noting that the conditions in our assumptions do not involve a convexity assumption on $h$, often imposed in the stochastic control literature. Also, although they appear to be involved, it is straightforward to check whether a given choice of the problem data satisfies the conditions of Assumption 2.2.

Example 2.3. If we choose

$$
b(x)=a x, \quad \sigma(x)=c, \quad h(x)=\zeta|x|^{p}, \quad k_{+}(x)=K^{+}, \quad k_{-}(x)=K^{-},
$$

for some constants $a \in \mathbb{R}, c \neq 0, \zeta, K^{+}, K^{-}>0$, and $p>1$, then Assumption 2.2 holds.

\section{The solution of the control problem}

With regard to the general theory of stochastic control, the solution of the control problem formulated in Section 2 can be obtained by finding a, sufficiently smooth for an application of Itô's formula, function $w$ and a constant $\lambda$ satisfying the following HamiltonJacobi-Bellman (HJB) equation:

$$
\min \left\{\frac{1}{2} \sigma^{2}(x) w^{\prime \prime}(x)+b(x) w^{\prime}(x)+h(x)-\lambda, k_{+}(x)+w^{\prime}(x), k_{-}(x)-w^{\prime}(x)\right\}=0 .
$$

If such a pair $(w, \lambda)$ exists, then, subject to suitable technical conditions, we expect the following statements to be true. Given any initial condition $x \in \mathbb{R}$,

$$
\lambda=\inf _{\mathbb{C}_{x} \in \mathscr{C}_{x}} J^{\mathrm{E}}\left(\mathbb{C}_{x}\right)=\inf _{\mathbb{C}_{x} \in \mathscr{C}_{x}} J^{\mathrm{P}}\left(\mathbb{C}_{x}\right),
$$

which reflects the fact that the optimal values of the performance criteria considered are independent of the system's initial condition. The set of all $x \in \mathbb{R}$ such that

$$
k_{+}(x)+w^{\prime}(x)=0
$$


defines the part of the state space in which the controller should exert minimal effort by increasing the process $\xi^{+}$so as to position and then reflect the state process at the closest boundary point of the set in the positive direction. Similarly, the set of all $x \in \mathbb{R}$ such that

$$
k_{-}(x)-w^{\prime}(x)=0
$$

defines the part of the state space where the controller should exert minimal effort by increasing the process $\xi^{-}$so as to position and then reflect the state process at the closest boundary point of the set in the negative direction. The interior of the set of all $x \in \mathbb{R}$ such that

$$
\frac{1}{2} \sigma^{2}(x) w^{\prime \prime}(x)+b(x) w^{\prime}(x)+h(x)-\lambda=0
$$

defines the part of the state space in which the controller should take no action.

With regard to the control problems considered, we conjecture that an optimal strategy is characterised by two points, $x_{-}<x_{+}$, and takes a form that can be described as follows. The controller exerts minimal effort so as to keep the state process within $\left[x_{-}, x_{+}\right]$. Accordingly, with the exception of a possible jump at time 0 , the process $\xi^{+}$is continuous and increases on the set of times when $X_{t}=x_{-}$in order to reflect the state process back into the no action region $] x_{-}, x_{+}\left[\right.$. Similarly, the process $\xi^{-}$can have a jump the size of $x-x_{+}$at time 0 so as to reposition the state process from its initial value $x$ to the boundary point $x_{+}$, if $x>x_{+}$, and then increases on the set of times when $X_{t}=x_{+}$.

Assuming that this strategy is indeed optimal, we need a system of appropriate equations to determine the free-boundary points $x_{-}, x_{+}$and the constant $\lambda$. To this end we conjecture that the so-called "smooth-pasting condition" holds, which, in the case of the singular control problem that we consider here, suggests that the function $w$ should be $C^{2}$, in particular, at the free boundary points $x_{-}$and $x_{+}$. We therefore look for a solution $(w, \lambda)$ to the HJB equation (3.1) such that

$$
\begin{gathered}
w^{\prime}(x)=-k_{+}(x), \quad w^{\prime \prime}(x)=-k_{+}^{\prime}(x), \quad \forall x \leq x_{-}, \\
\left.\frac{1}{2} \sigma^{2}(x) w^{\prime \prime}(x)+b(x) w^{\prime}(x)+h(x)-\lambda=0, \quad \text { for } x \in\right] x_{-}, x_{+}[ \\
w^{\prime}(x)=k_{-}(x), \quad w^{\prime \prime}(x)=k_{-}^{\prime}(x), \quad \forall x \geq x_{+} .
\end{gathered}
$$

The four equations resulting from (3.6) and (3.8) for $x=x_{-}$and for $x=x_{+}$, respectively, suggest that we should consider a fourth parameter. To determine such a parameter, we observe that the strict positivity of $k_{+}, k_{-}$and the fact that $w^{\prime}$ is continuous imply that $w$ should have a local minimum inside $] x_{-}, x_{+}$, denoted by $x_{0}$, so that $w^{\prime}\left(x_{0}\right)=0$. With regard to this observation, we note that the solution to the ODE (3.5) with initial condition $w^{\prime}\left(x_{0}\right)=0$ is given by

$$
w^{\prime}(x)=p_{x_{0}}^{\prime}(x) \int_{x_{0}}^{x}[\lambda-h(s)] m_{x_{0}}(d s), \quad x \in \mathbb{R},
$$

where $p_{x_{0}}$ and $m_{x_{0}}$ are the scale function and the speed measure of the uncontrolled diffusion (2.11), defined by (2.12) and (2.13), respectively. It follows that we need to determine 
the four parameters $x_{-}<x_{0}<x_{+}$and $\lambda$ that solve the nonlinear system of equations

$$
\begin{gathered}
g\left(x_{+}, \lambda, x_{0}\right)=k_{-}\left(x_{+}\right), \quad \frac{\partial g}{\partial x}\left(x_{+}, \lambda, x_{0}\right)=k_{-}^{\prime}\left(x_{+}\right), \\
g\left(x_{-}, \lambda, x_{0}\right)=-k_{+}\left(x_{-}\right), \quad \frac{\partial g}{\partial x}\left(x_{-}, \lambda, x_{0}\right)=-k_{+}^{\prime}\left(x_{-}\right),
\end{gathered}
$$

where $g$ is defined by

$$
g\left(x, \lambda, x_{0}\right)=p_{x_{0}}^{\prime}(x) \int_{x_{0}}^{x}[\lambda-h(s)] m_{x_{0}}(d s), \quad \text { for } x, \lambda, x_{0} \in \mathbb{R} .
$$

The following result, the proof of which is developed in the Appendix, is concerned with showing that the heuristic considerations above indeed provide a solution to the HJB equation (3.1).

Lemma 3.1. Suppose that Assumption 2.2 holds. The system of (3.10), where $g$ is defined by (3.11), has a solution $\left(x_{-}, x_{0}, x_{+}, \lambda\right)$ such that $x_{-}<x_{0}<x_{+}$, and, if $w$ is a function satisfying (3.9) inside the interval ] $x_{-}, x_{+}[$and is given by (3.6) and (3.8) in the complement of ]$x_{-}, x_{+}\left[\right.$, then $w$ is $C^{2}$ and the pair $(w, \lambda)$ is a solution to the HJB equation (3.1).

We can now prove the main result of the paper that concerns the optimisation of the ergodic expected criterion.

THEOREM 3.2. Consider the stochastic control problem formulated in Section 2 that aims at the minimisation of the long-term average expected criterion defined by (2.2)-(2.3). Suppose that Assumption 2.2 holds, and let the constants $x_{-}, x_{0}, x_{+}, \lambda$ be as in Lemma 3.1. Then, given any initial condition $x \in \mathbb{R}$,

$$
\lambda=\inf _{\mathbb{C}_{x} \in \mathscr{C}_{x}} J^{\mathrm{E}}\left(\mathbb{C}_{x}\right),
$$

and the points $x_{-}, x_{+}$determine the optimal strategy that has been discussed qualitatively above.

Proof. Throughout the proof, we fix the solution $(w, \lambda)$ to the HJB equation (3.1) that is constructed in Lemma 3.1. We also fix an initial condition $x \in \mathbb{R}$.

Consider any admissible control $\mathbb{C}_{x} \in \mathscr{C}_{x}$ such that $J^{\mathrm{E}}\left(\mathbb{C}_{x}\right)<\infty$. Using Itô's formula for general semimartingales, we obtain

$$
\begin{aligned}
w\left(X_{T+}\right)= & w(x)+\int_{0}^{T}\left[\frac{1}{2} \sigma^{2}\left(X_{s}\right) w^{\prime \prime}\left(X_{s}\right)+b\left(X_{s}\right) w^{\prime}\left(X_{s}\right)\right] d s+\int_{[0, T]} w^{\prime}\left(X_{s}\right) d \xi_{s} \\
& +\int_{0}^{T} \sigma\left(X_{s}\right) w^{\prime}\left(X_{s}\right) d W_{s}+\sum_{s \in[0, T]}\left[w\left(X_{s+}\right)-w\left(X_{s}\right)-w^{\prime}\left(X_{s}\right) \Delta X_{s}\right] \\
= & w(x)+\int_{0}^{T}\left[\frac{1}{2} \sigma^{2}\left(X_{s}\right) w^{\prime \prime}\left(X_{s}\right)+b\left(X_{s}\right) w^{\prime}\left(X_{s}\right)\right] d s+\int_{0}^{T} \sigma\left(X_{s}\right) w^{\prime}\left(X_{s}\right) d W_{s} \\
& +\int_{[0, T]} w^{\prime}\left(X_{s}\right) d\left(\xi^{+}\right)_{s}^{c}-\int_{[0, T]} w^{\prime}\left(X_{s}\right) d\left(\xi^{-}\right)_{s}^{c}+\sum_{s \in[0, T]}\left[w\left(X_{s+}\right)-w\left(X_{s}\right)\right],
\end{aligned}
$$


the second equality following because $\xi_{s}=\xi_{s}^{+}-\xi_{s}^{-}$and the jumps of $X$ coincide with those of $\xi$. Here $\left(\xi^{+}\right)^{c}$ and $\left(\xi^{-}\right)^{c}$ are the continuous parts of the processes $\xi^{+}$and $\xi^{-}$, respectively. Now, given any time $s \geq 0$,

$$
w\left(X_{s+}\right)-w\left(X_{s}\right)=\int_{0}^{\Delta\left(\xi^{+}\right)_{s}} w^{\prime}\left(X_{s}+r\right) d r-\int_{0}^{\Delta\left(\xi^{-}\right)_{s}} w^{\prime}\left(X_{s}-r\right) d r
$$

Using this observation and the definitions (1.4), we can see that (3.13) implies

$$
\begin{aligned}
I_{T}\left(\mathbb{C}_{x}\right):= & \int_{0}^{T} h\left(X_{s}\right) d s+\int_{[0, T]} k_{+}\left(X_{s}\right) \oplus d \xi_{s}^{+}+\int_{[0, T]} k_{-}\left(X_{s}\right) \ominus d \xi_{s}^{-} \\
= & \lambda T+w(x)-w\left(X_{T}\right)+\int_{0}^{T} \sigma\left(X_{s}\right) w^{\prime}\left(X_{s}\right) d W_{s} \\
& +\int_{0}^{T}\left[\frac{1}{2} \sigma^{2}\left(X_{s}\right) w^{\prime \prime}\left(X_{s}\right)+b\left(X_{s}\right) w^{\prime}\left(X_{s}\right)+h\left(X_{s}\right)-\lambda\right] d s \\
& +\int_{0}^{T}\left[k_{+}\left(X_{s}\right)+w^{\prime}\left(X_{s}\right)\right] d\left(\xi^{+}\right)_{s}^{c}+\int_{0}^{T}\left[k_{-}\left(X_{s}\right)-w^{\prime}\left(X_{s}\right)\right] d\left(\xi^{-}\right)_{s}^{c} \\
& +\sum_{s \in[0, T]} \int_{0}^{\Delta\left(\xi^{+}\right)_{s}}\left[k_{+}\left(X_{s}+r\right)+w^{\prime}\left(X_{s}+r\right)\right] d r \\
& +\sum_{s \in[0, T]} \int_{0}^{\Delta\left(\xi^{-}\right)_{s}}\left[k_{-}\left(X_{s}-r\right)-w^{\prime}\left(X_{s}-r\right)\right] d r .
\end{aligned}
$$

Since the pair $(w, \lambda)$ satisfies the HJB equation (3.1), it follows that

$$
I_{T}\left(\mathbb{C}_{x}\right) \geq \lambda T+w(x)-w\left(X_{T}\right)+\int_{0}^{T} \sigma\left(X_{s}\right) w^{\prime}\left(X_{s}\right) d W_{s}
$$

By construction, $w$ is $C^{2}, w^{\prime}(x)=k_{-}(x)$, for all $x \geq x_{+}$, and $w^{\prime}(x)=-k_{+}(x)$, for all $x \leq x_{-}$. Therefore, in view of (2.8) in Assumption 2.2, there exists a constant $C_{4}>0$ such that

$$
w(x) \leq C_{4}(1+|x|), \quad\left|w^{\prime}(x)\right| \leq C_{4}, \quad \forall x \in \mathbb{R} .
$$

For such a choice of $C_{4},(3.16)$ yields

$$
I_{T}\left(\mathbb{C}_{x}\right) \geq \lambda T+w(x)-C_{4}-C_{4}\left|X_{T}\right|+\int_{0}^{T} \sigma\left(X_{s}\right) w^{\prime}\left(X_{s}\right) d W_{s}
$$

Now, with respect to the positivity of $k_{+}$and $k_{-}$, and Assumption 2.2(b),

$$
\infty>J^{\mathrm{E}}\left(\mathbb{C}_{x}\right) \geq \limsup _{T \rightarrow \infty} \frac{1}{T} E\left[\int_{0}^{T} h\left(X_{s}\right) d s\right] \geq-C_{2}+C_{2} \limsup _{T \rightarrow \infty} \frac{1}{T} E\left[\int_{0}^{T}\left|X_{s}\right| d s\right] .
$$


These inequalities imply

$$
\begin{gathered}
E\left[\int_{0}^{T}\left|X_{s}\right| d s\right]<\infty, \quad \forall T>0, \\
\liminf _{T \rightarrow \infty} \frac{1}{T} E\left[\left|X_{T}\right|\right]=0 .
\end{gathered}
$$

To see (3.21), suppose that $\liminf _{T \rightarrow \infty} T^{-1} E\left[\left|X_{T}\right|\right]>\varepsilon>0$. This implies that there exists $T_{1} \geq 0$ such that $E\left[\left|X_{s}\right|\right]>\varepsilon s / 2$, for all $s \geq T_{1}$. It follows that

$$
\limsup _{T \rightarrow \infty} \frac{1}{T} E\left[\int_{0}^{T}\left|X_{s}\right| d s\right] \geq \underset{T \rightarrow \infty}{\limsup } \frac{1}{T} \int_{T_{1}}^{T} \frac{\varepsilon s}{2} d s=\infty
$$

which contradicts (3.19).

With regard to (2.5) in Assumption 2.2(a), the second inequality in (3.17) and (3.20), we calculate

$$
E\left[\int_{0}^{T}\left[\sigma\left(X_{s}\right) w^{\prime}\left(X_{s}\right)\right]^{2} d s\right] \leq C_{4}^{2} C_{1}\left(T+E\left[\int_{0}^{T}\left|X_{s}\right| d s\right]\right)<\infty, \quad \forall T>0
$$

which proves that the stochastic integral in (3.18) is a square integrable martingale, and therefore has zero expectation. In view of this observation, we can take expectations in (3.18) and divide by $T$ to obtain

$$
\frac{1}{T} E\left[I_{T}\left(\mathbb{C}_{x}\right)\right] \geq \lambda+\frac{w(x)}{T}-\frac{C_{4}}{T}-\frac{C_{4}}{T} E\left[\left|X_{T}\right|\right]
$$

In view of (3.21) and the definition of $I_{T}\left(\mathbb{C}_{x}\right)$ in (3.15), we can pass to the limit $T \rightarrow \infty$ to obtain $J^{\mathrm{E}}\left(\mathbb{C}_{x}\right) \geq \lambda$.

To prove the reverse inequality, suppose that we can find a control

$$
\widehat{\mathbb{C}}_{x}=\left(\widehat{\Omega}, \hat{\mathscr{F}}, \hat{\mathscr{F}}_{t}, \hat{P}, \widehat{W}, \hat{\xi}, \hat{X}, \hat{\tau}\right) \in \mathscr{C}_{x}
$$

such that

$$
\begin{gathered}
\hat{X}_{t} \in\left[x_{-}, x_{+}\right], \quad \forall t>0, \hat{P} \text {-a.s. } \\
\Delta \hat{\xi}_{0}=\left(x_{-}-x\right)^{+}-\left(x-x_{+}\right)^{+}, \quad \hat{P} \text {-a.s. } \\
\int_{] 0, \infty[} \mathbf{1}_{\left.x_{-,}, x_{+}\right]}\left(\hat{X}_{s}\right) d \hat{\xi}_{s}^{+}=0, \quad \int_{] 0, \infty[} \mathbf{1}_{\left[x_{-}, x_{+}\right.}\left(\hat{X}_{s}\right) d \hat{\xi}_{s}^{-}=0, \quad \hat{P} \text {-a.s. }
\end{gathered}
$$

Plainly, (3.26) implies that $\hat{X}$ is nonexplosive, so that $\hat{\tau}=\infty, \hat{P}$-a.s. Also, with regard to 
the construction of $w$, we can see that, for such a choice of a control, (3.15) implies

$$
I_{T}\left(\widehat{\mathbb{C}}_{x}\right)=\lambda T+w(x)-w\left(\widehat{X}_{T}\right)+\int_{0}^{T} \sigma\left(\widehat{X}_{s}\right) w^{\prime}\left(\widehat{X}_{s}\right) d \widehat{W}_{s}
$$

Now, (2.5) in Assumption 2.2, (3.17) and (3.26) imply

$$
\widehat{E}\left[\int_{0}^{T}\left[\sigma\left(\hat{X}_{s}\right) w^{\prime}\left(\hat{X}_{s}\right)\right]^{2} d s\right] \leq C_{4}^{2} C_{1}\left(1+\left|x_{-}\right| \vee\left|x_{+}\right|\right) T<\infty, \quad \forall T>0,
$$

which proves that the stochastic integral in (3.29) is a square integrable martingale, and

$$
\lim _{T \rightarrow \infty} \frac{1}{T} \hat{E}\left[\left|w\left(\hat{X}_{T}\right)\right|\right] \leq \lim _{T \rightarrow \infty} \frac{1}{T} C_{4}\left(1+\left|x_{-}\right| \vee\left|x_{+}\right|\right)=0 .
$$

It follows that

$$
\lim _{T \rightarrow \infty} \frac{1}{T} \hat{E}\left[I_{T}\left(\widehat{\mathbb{C}}_{x}\right)\right]=\lambda
$$

which proves that $J^{\mathrm{E}}\left(\widehat{\mathbb{C}}_{x}\right)=\lambda$, and establishes (3.12).

Finally, we note that a control $\widehat{\mathbb{C}}_{x}$ satisfying (3.26)-(3.28), which is optimal, can be constructed as in [16].

The following result is concerned with the solution to the optimisation problem considered with the ergodic pathwise criterion.

THEOREM 3.3. Consider the stochastic control problem formulated in Section 2 that aims at the minimisation of the long-term average pathwise criterion defined by (2.4). Suppose that Assumption 2.2 holds, and let the constants $x_{-}, x_{0}, x_{+}, \lambda$ be as in Lemma 3.1. Then, given any initial condition $x \in \mathbb{R}$,

$$
\lambda=\inf _{\mathbb{C}_{x} \in \mathscr{C}_{x}} J^{\mathrm{P}}\left(\mathbb{C}_{x}\right)
$$

and the points $x_{-}, x_{+}$determine the optimal strategy.

Proof. Throughout the proof, we fix the solution $(w, \lambda)$ to the HJB equation $(3.1)$ that is constructed in Lemma 3.1. We also fix an initial condition $x \in \mathbb{R}$.

Consider any admissible control $\mathbb{C}_{x} \in \mathscr{C}_{x}$. Using the same arguments as the ones that established (3.18) in the proof of Theorem 3.2 above, we can show that

$$
\begin{aligned}
I_{T}\left(\mathbb{C}_{x}\right) \mathbf{1}_{\{T<\tau\}} & :=\left(\int_{0}^{T} h\left(X_{s}\right) d s+\int_{[0, T]} k_{+}\left(X_{s}\right) \oplus d \xi_{s}^{+}+\int_{[0, T]} k_{-}\left(X_{s}\right) \ominus d \xi_{s}^{-}\right) \mathbf{1}_{\{T<\tau\}} \\
& \geq\left(\lambda T+w(x)-C_{4}-C_{4}\left|X_{T}\right|+M_{T}\right) \mathbf{1}_{\{T<\tau\}},
\end{aligned}
$$

where

$$
M_{T}=\int_{0}^{T} \sigma\left(X_{s}\right) w^{\prime}\left(X_{s}\right) d W_{s}
$$


With regard to the positivity of $k_{+}$and $k_{-}$, and Assumption 2.2(b), we can see that

$$
\begin{aligned}
\infty & >J^{\mathrm{P}}\left(\mathbb{C}_{x}\right) \mathbf{1}_{\left\{J^{\mathrm{P}}\left(\mathbb{C}_{x}\right)<\infty\right\}} \geq\left(\limsup _{T \rightarrow \infty} \frac{1}{T} \int_{0}^{T} h\left(X_{s}\right) d s\right) \mathbf{1}_{\left\{J^{\mathrm{P}}\left(\mathbb{C}_{x}\right)<\infty\right\}} \\
& \geq C_{2}\left(-1+\limsup _{T \rightarrow \infty} \frac{1}{T} \int_{0}^{T}\left|X_{s}\right| d s\right) \mathbf{1}_{\left\{J^{\mathrm{P}}\left(\mathbb{C}_{x}\right)<\infty\right\}} .
\end{aligned}
$$

By appealing to arguments similar to the ones that established (3.21), we can see that these inequalities imply

$$
\liminf _{T \rightarrow \infty} \frac{1}{T}\left|X_{T}\right| \mathbf{1}_{\left\{J^{\mathrm{P}}\left(\mathbb{C}_{x}\right)<\infty\right\}}=0 .
$$

Also, they imply that there exists a real-valued random variable $Z$ and a random time $\tau_{Z}$ such that

$$
\left(\frac{1}{T} \int_{0}^{T}\left|X_{s}\right| d s\right) \mathbf{1}_{\left\{J^{\mathrm{P}}\left(\mathbb{C}_{x}\right)<\infty\right\}} \leq Z \mathbf{1}_{\left\{J^{\mathrm{P}}\left(\mathbb{C}_{x}\right)<\infty\right\}}, \quad \forall T \geq \tau_{Z} .
$$

In view of (2.5) in Assumption 2.2(a) and the second estimate in (3.17), it follows that

$$
\begin{aligned}
\langle M\rangle_{T} \mathbf{1}_{\left\{J^{\mathrm{P}}\left(\mathbb{C}_{x}\right)<\infty\right\}} & \leq C_{4}^{2} C_{1}\left(1+\frac{1}{T} \int_{0}^{T}\left|X_{s}\right| d s\right) T \mathbf{1}_{\left\{J^{\mathrm{P}}\left(\mathbb{C}_{x}\right)<\infty\right\}} \\
& \leq C_{4}^{2} C_{1}(1+Z) T \mathbf{1}_{\left\{J^{\mathrm{P}}\left(\mathbb{C}_{x}\right)<\infty\right\}}, \quad \forall T \geq \tau_{Z},
\end{aligned}
$$

where $\langle M\rangle$ is the quadratic variation process of the local martingale $M$ defined in (3.35).

Now, with regard to the Dambis, Dubins, and Schwarz theorem (e.g., see Revuz and Yor [14, Theorem V.1.7]), there exists a standard, one-dimensional Brownian motion $B$ defined on a possible extension of $(\Omega, \mathscr{F}, P)$ such that

$$
\langle M\rangle_{T} \mathbf{1}_{\{T \leq \tau\}}=B_{\langle M\rangle_{T}} \mathbf{1}_{\{T \leq \tau\}} .
$$

In view of this representation, the observation that

$$
\left\{J^{\mathrm{P}}\left(\mathbb{C}_{x}\right)<\infty\right\} \subseteq\{\tau=\infty\},
$$

(3.39), and the fact that $\lim _{T \rightarrow \infty} B_{T} / T=0$, we can see that

$$
\begin{aligned}
\lim _{T \rightarrow \infty} & \frac{1}{T}\left|M_{T}\right| \mathbf{1}_{\left\{J^{\mathrm{P}}\left(\mathbb{C}_{x}\right)<\infty\right\} \cap\left\{\langle M\rangle_{\infty}=\infty\right\}} \\
& =\lim _{T \rightarrow \infty} \frac{1}{T}\left|B_{\langle M\rangle_{T}}\right| \mathbf{1}_{\left\{J^{\mathrm{P}}\left(\mathbb{C}_{x}\right)<\infty\right\} \cap\left\{\langle M\rangle_{\infty}=\infty\right\}} \\
& \leq C_{4}^{2} C_{1}(1+Z) \lim _{T \rightarrow \infty} \frac{1}{\langle M\rangle_{T}}\left|B_{\langle M\rangle_{T}}\right| \mathbf{1}_{\left\{\mathrm{J}^{\mathrm{P}}\left(\mathbb{C}_{x}\right)<\infty\right\} \cap\left\{\langle M\rangle_{\infty}=\infty\right\}}=0 .
\end{aligned}
$$

Furthermore, since a continuous local martingale $M$ converges in $\mathbb{R}, P$-a.s., on the event $\left\{\langle M\rangle_{\infty}<\infty\right\}$ (e.g., see Revuz and Yor [14, Proposition IV.1.26]),

$$
\lim _{T \rightarrow \infty} \frac{1}{T}\left|M_{T}\right| \mathbf{1}_{\left\{J^{\mathrm{P}}\left(\mathbb{C}_{x}\right)<\infty\right\} \cap\left\{\langle M\rangle_{\infty}<\infty\right\}}=0 .
$$


However, combining (3.42) and (3.43) with (3.34), (3.37), and (3.41), we can see that

$$
J^{\mathrm{P}}\left(\mathbb{C}_{x}\right) \equiv \limsup _{T \rightarrow \infty} \frac{1}{T} I_{T}\left(\mathbb{C}_{x}\right) \geq \lambda
$$

To prove the reverse inequality, consider the control $\widehat{\mathbb{C}}_{x} \in \mathscr{C}_{x}$ satisfying (3.26)-(3.28), which is associated with $\hat{\tau}=\infty$ and

$$
I_{T}\left(\widehat{\mathbb{C}}_{x}\right)=\lambda T+w(x)-w\left(\widehat{X}_{T}\right)+\widehat{M}_{T}
$$

where $I_{T}$ and $\widehat{M}$ are defined as in (3.34) and (3.35), respectively, (see also (3.29)). Since $\hat{X}_{t} \in\left[x_{-}, x_{+}\right]$, for all $t \geq 0, \hat{P}$-a.s., (2.5) in Assumption 2.2(a) and (3.17) imply

$$
\left|w\left(X_{T}\right)\right| \leq C_{4}\left(1+\left|x_{-}\right| \vee\left|x_{+}\right|\right), \quad\langle M\rangle_{T} \leq C_{4}^{2} C_{1}\left(1+\left|x_{-}\right| \vee\left|x_{+}\right|\right) T, \quad \forall T \geq 0 .
$$

However, in light of these inequalities and an argument such as the one establishing (3.42) and (3.43) above, we can see that $J^{\mathrm{P}}\left(\widehat{\mathbb{C}}_{x}\right) \equiv \lim _{T \rightarrow \infty} I_{T}\left(\hat{\mathbb{C}}_{x}\right) / T=\lambda$, and the proof is complete.

\section{Appendix}

To prepare the grounds for proving Lemma 3.1, we note that the calculation

$$
\frac{d}{d x} \frac{k_{ \pm}(x)}{p_{x_{0}}^{\prime}(x)}=\frac{2}{\sigma^{2}(x) p_{x_{0}}^{\prime}(x)}\left[\frac{1}{2} \sigma^{2}(x) k_{ \pm}^{\prime}(x)+b(x) k_{ \pm}(x)\right]
$$

the definition (3.11) of $g$, and the definition (2.13) of the speed measure $m_{x_{0}}$ imply

$g\left(x, \lambda, x_{0}\right)-k_{ \pm}(x)=p_{x_{0}}^{\prime}(x)\left[\int_{x_{0}}^{x}\left[\lambda-\frac{1}{2} \sigma^{2}(s) k_{ \pm}^{\prime}(s)-b(s) k_{ \pm}(s)-h(s)\right] m_{x_{0}}(d s)-k_{ \pm}\left(x_{0}\right)\right]$.

Also, we calculate

$$
\begin{gathered}
\frac{\partial g}{\partial x}\left(x, \lambda, x_{0}\right)=-\frac{2}{\sigma^{2}(x)}\left[b(x) g\left(x, \lambda, x_{0}\right)+h(x)-\lambda\right] \\
\frac{\partial g}{\partial \lambda}\left(x, \lambda, x_{0}\right)=\operatorname{sgn}\left(x-x_{0}\right) p_{x_{0}}^{\prime}(x) m_{x_{0}}\left(\left[x_{0} \wedge x, x_{0} \vee x\right]\right), \\
\frac{\partial g}{\partial x_{0}}\left(x, \lambda, x_{0}\right)=-\frac{2\left[\lambda-h\left(x_{0}\right)\right]}{\sigma^{2}\left(x_{0}\right)} p_{x_{0}}^{\prime}(x)
\end{gathered}
$$

and we observe that (A.4) implies

$$
\lim _{\lambda \rightarrow \infty} g\left(x, \lambda, x_{0}\right)= \begin{cases}\infty, & \text { for } x>x_{0} \\ -\infty, & \text { for } x<x_{0}\end{cases}
$$


In view of the structure and the continuity of $g$, the positivity of $h$, and the continuity and strict positivity of $k_{-}$and $k_{+}$, this observation implies

$$
\begin{aligned}
& \left.\Lambda_{+}\left(x_{0}\right):=\inf \left\{\lambda \in \mathbb{R} \mid \sup _{x \geq x_{0}}\left[g\left(x, \lambda, x_{0}\right)-k_{-}(x)\right] \geq 0\right\} \in\right] 0, \infty\left[, \quad \forall x_{0} \in \mathbb{R},\right. \\
& \left.\Lambda_{-}\left(x_{0}\right):=\inf \left\{\lambda \in \mathbb{R} \mid \inf _{x \leq x_{0}}\left[g\left(x, \lambda, x_{0}\right)+k_{+}(x)\right] \leq 0\right\} \in\right] 0, \infty\left[, \quad \forall x_{0} \in \mathbb{R} .\right.
\end{aligned}
$$

Given these definitions we can prove the following result.

Lemma A.1. Given $x_{0} \in \mathbb{R}$, let $\Lambda_{+}\left(x_{0}\right)$ and $\Lambda_{-}\left(x_{0}\right)$ be defined by (A.7) and (A.8), respectively. The equations $g\left(x, \Lambda_{ \pm}\left(x_{0}\right), x_{0}\right)= \pm k_{\mp}(x)$ determine uniquely two continuous functions $\chi_{ \pm}$such that

$$
\begin{gathered}
-\infty<\chi_{-}\left(x_{0}\right)<x_{0}<\chi_{+}\left(x_{0}\right)<\infty, \\
g\left(\chi_{+}\left(x_{0}\right), \Lambda_{+}\left(x_{0}\right), x_{0}\right)=k_{-}\left(\chi_{+}\left(x_{0}\right)\right), \\
\frac{\partial g}{\partial x}\left(\chi_{+}\left(x_{0}\right), \Lambda_{+}\left(x_{0}\right), x_{0}\right)=k_{-}^{\prime}\left(\chi_{+}\left(x_{0}\right)\right), \\
g\left(\chi_{-}\left(x_{0}\right), \Lambda_{-}\left(x_{0}\right), x_{0}\right)=-k_{+}\left(\chi_{-}\left(x_{0}\right)\right), \\
\frac{\partial g}{\partial x}\left(\chi_{-}\left(x_{0}\right), \Lambda_{-}\left(x_{0}\right), x_{0}\right)=-k_{+}^{\prime}\left(\chi_{-}\left(x_{0}\right)\right),
\end{gathered}
$$

for all $x_{0} \in \mathbb{R}$. These functions satisfy

$$
\begin{array}{cl}
g\left(x, \Lambda_{+}\left(x_{0}\right), x_{0}\right)<k_{-}(x), & \forall x \in\left[x_{0}, \chi_{+}\left(x_{0}\right)[\right. \\
g\left(x, \Lambda_{-}\left(x_{0}\right), x_{0}\right)>-k_{+}(x), & \left.\forall x \in] \chi_{-}\left(x_{0}\right), x_{0}\right], \\
\chi_{-}\left(x_{0}\right)<\alpha_{-}, & a_{+}<\chi_{+}\left(x_{0}\right),
\end{array}
$$

for all $x_{0} \in \mathbb{R}$, where $\alpha_{-}$and $a_{+}$are as in Assumptions 2.2(e) and 2.2(f), respectively. Morover, there exists a unique point $\tilde{x}_{0} \in \mathbb{R}$ such that

$$
\tilde{\lambda}:=\Lambda_{+}\left(\tilde{x}_{0}\right)=\Lambda_{-}\left(\tilde{x}_{0}\right), \quad h\left(\tilde{x}_{0}\right)<\tilde{\lambda}
$$

Proof. Fix any $x_{0} \in \mathbb{R}$. With regard to the definition (A.7) of $\Lambda_{+}\left(x_{0}\right)$, we define

$$
\chi_{+}\left(x_{0}\right)=\inf \left\{x>x_{0} \mid g\left(x, \Lambda_{+}\left(x_{0}\right), x_{0}\right)=k_{-}(x)\right\}>x_{0},
$$

with the usual convention $\inf \varnothing=\infty$. Here, the strict inequality follows thanks to the strict positivity of $k_{-}$and the fact that $g\left(x_{0}, \lambda, x_{0}\right)=0$, for all $\lambda \in \mathbb{R}$. If we assume that $\chi_{+}\left(x_{0}\right)=\infty$, then the continuity of the functions $g, k_{-}$, the boundedness of $k_{-}$and the definition (A.7) of $\Lambda_{+}\left(x_{0}\right)$ imply

$$
\left|g\left(x, \Lambda_{+}\left(x_{0}\right), x_{0}\right)\right| \leq C_{5}, \quad \forall x \geq x_{0},
$$


for some constant $C_{5}>0$, and

$$
\lim _{x \rightarrow \infty}\left[g\left(x, \Lambda_{+}\left(x_{0}\right), x_{0}\right)-k_{-}(x)\right]=0 .
$$

With regard to (A.3) and (A.19), it follows that

$$
\lim _{x \rightarrow \infty} \frac{\partial g}{\partial x}\left(x, \Lambda_{+}\left(x_{0}\right), x_{0}\right) \leq \lim _{x \rightarrow \infty} \frac{2}{\sigma^{2}(x)}\left[C_{5}|b(x)|-h(x)+\Lambda_{+}\left(x_{0}\right)\right]=-\infty
$$

the last equality following thanks to Assumptions 2.2(b) and 2.2(c). This calculation and the boundedness of $k_{-}$contradict (A.20), which proves that $\chi_{+}\left(x_{0}\right)$ is finite. However, this conclusion and the fact that

$$
\chi_{+}\left(x_{0}\right) \text { is a local maximum of the } C^{1} \text { function } x \longmapsto g\left(x, \Lambda_{+}\left(x_{0}\right), x_{0}\right)-k_{-}(x) \text {, }
$$

which follows from the definitions (A.7) and (A.18), imply that (A.10), (A.11), and (A.14) are satisfied.

With regard to Assumption 2.2(f) and the strict positivity of $\Lambda_{+}\left(x_{0}\right)$, there exist two points $y_{-}$and $y_{+}$such that

$$
\begin{gathered}
y_{-}<a_{-} \leq a_{+}<y_{+}, \\
-\frac{2}{\sigma^{2}(x)}\left[\frac{1}{2} \sigma^{2}(x) k_{-}^{\prime}(x)+b(x) k_{-}(x)+h(x)-\Lambda_{+}\left(x_{0}\right)\right] \begin{cases}<0, & \text { for } x<y_{-}, \\
>0, & \text { for } x \in] y_{-}, y_{+}[ \\
<0, & \text { for } x>y_{+} .\end{cases}
\end{gathered}
$$

Combining this observation with the identity

$$
\frac{1}{2} \sigma^{2}\left(\chi_{+}\left(x_{0}\right)\right) k_{-}^{\prime}\left(\chi_{+}\left(x_{0}\right)\right)+b\left(\chi_{+}\left(x_{0}\right)\right) k_{-}\left(\chi_{+}\left(x_{0}\right)\right)+h\left(\chi_{+}\left(x_{0}\right)\right)-\Lambda_{+}\left(x_{0}\right)=0,
$$

which follows from (A.3) and (A.10)-(A.11), we can see that either $\chi_{+}\left(x_{0}\right)=y_{-}$or $\chi_{+}\left(x_{0}\right)=y_{+}$. Now, suppose that $\chi_{+}\left(x_{0}\right)=y_{-}$. In view of (A.4), (A.22), and the continuity of $g$ and $k_{-}$, such an assumption implies that there exist $\varepsilon>0$, sufficiently small, and points $z_{1}, z_{2}$ such that

$$
\begin{gathered}
x_{0}<z_{1}<y_{-}<z_{2}<y_{+}, \\
g\left(z_{1}, \Lambda_{+}\left(x_{0}\right)+\varepsilon, x_{0}\right)=k_{-}\left(z_{1}\right), \quad g\left(z_{2}, \Lambda_{+}\left(x_{0}\right)+\varepsilon, x_{0}\right)=k_{-}\left(z_{2}\right), \\
\frac{\partial}{\partial x}\left[g\left(x, \Lambda_{+}\left(x_{0}\right)+\varepsilon, x_{0}\right)-k_{-}(x)\right] \begin{cases}>0, & \text { for } x=z_{1}, \\
<0, & \text { for } x=z_{2} .\end{cases}
\end{gathered}
$$


Combining (A.27) and (A.28) with (A.3), we can see that

$$
\begin{gathered}
-\frac{2}{\sigma^{2}\left(z_{2}\right)}\left[\frac{1}{2} \sigma^{2}\left(z_{2}\right) k_{-}^{\prime}\left(z_{2}\right)+b\left(z_{2}\right) k_{-}\left(z_{2}\right)+h\left(z_{2}\right)-\Lambda_{+}\left(x_{0}\right)\right] \\
<-\frac{2}{\sigma^{2}\left(z_{2}\right)}\left[\frac{1}{2} \sigma^{2}\left(z_{2}\right) \frac{\partial g}{\partial x}\left(z_{2}, \Lambda_{+}\left(x_{0}\right)+\varepsilon, x_{0}\right)+b\left(z_{2}\right) g\left(z_{2}, \Lambda_{+}\left(x_{0}\right)+\varepsilon, x_{0}\right)\right. \\
\left.+h\left(z_{2}\right)-\Lambda_{+}\left(x_{0}\right)\right]=-\frac{2}{\sigma^{2}\left(z_{2}\right)} \varepsilon<0,
\end{gathered}
$$

which contradicts (A.24) because $\left.z_{2} \in\right] y_{-}, y_{+}[$(see (A.26)). However, this proves that $\chi_{+}\left(x_{0}\right)=y_{+}$, which, in view of (A.23), implies the corresponding claim in (A.16). Also, arguing by contradiction, we can use similar methods to show that $\chi_{+}\left(x_{0}\right)$ is unique.

To proceed further, we note that Assumption 2.2(b), the strict positivity of $k_{-}$, and the definition (3.11) of $g$ imply that $\Lambda_{+}\left(x_{0}\right)>h\left(x_{0}\right)$, for all $x_{0} \geq 0$. In particular,

$$
A_{-}:=\inf \left\{x_{0} \in \mathbb{R} \mid \Lambda_{+}\left(x_{0}\right)>h\left(x_{0}\right)\right\} \in[-\infty, 0[\text {. }
$$

In view of this observation, (A.5) and (A.14), we can see that, given any $x_{0} \geq A_{-}$,

$$
\left.g\left(x, \Lambda_{+}\left(x_{0}\right), \bar{x}_{0}\right)<k_{-}(x), \quad \forall x \in\left[\bar{x}_{0}, \chi_{+}\left(x_{0}\right)\right], \bar{x}_{0} \in\right] x_{0}, \chi_{+}\left(x_{0}\right)[
$$

Also, we note that (A.25), (A.16), and Assumption 2.2(f) imply that, if $\Lambda_{+}$is increasing (resp., decreasing) at some point $x_{0} \in \mathbb{R}$, then $\chi_{+}$is increasing (resp., decreasing) at $x_{0}$. However, combining this observation with (A.31), the definition (3.11) of $g$ and the strict positivity of $k_{-}$, we can see that

$$
\Lambda_{+}\left(x_{0}\right)<\Lambda_{+}\left(\bar{x}_{0}\right), \quad \chi_{+}\left(x_{0}\right)<\chi_{+}\left(\bar{x}_{0}\right), \quad \forall A_{-} \leq x_{0}<\bar{x}_{0}
$$

that is, $\Lambda_{+}$and $\chi_{+}$both are strictly increasing in $] A_{-}, \infty[$.

Now, we argue by contradiction and we assume that $\Lambda_{+}$is discontinuous at some point $x_{0}^{*}>A_{-}$, so that

$$
\begin{gathered}
\underline{\Lambda}_{+}\left(x_{0}^{*}\right):=\lim _{x_{0} \uparrow x_{0}^{*}} \Lambda_{+}\left(x_{0}\right)<\lim _{x_{0} \downarrow x_{0}^{*}} \Lambda_{+}\left(x_{0}\right)=: \bar{\Lambda}_{+}\left(x_{0}^{*}\right), \\
\underline{\chi}_{+}\left(x_{0}^{*}\right):=\lim _{x_{0} \uparrow x_{0}^{*}} \chi_{+}\left(x_{0}\right) \leq \lim _{x_{0} \downarrow x_{0}^{*}} \chi_{+}\left(x_{0}\right)=: \bar{\chi}_{+}\left(x_{0}^{*}\right) .
\end{gathered}
$$

Since the rest of the associated functions are continuous, (A.2) and (A.10) imply

$$
\begin{aligned}
0 & =\int_{x_{0}^{*}}^{\underline{x}_{+}\left(x_{0}^{*}\right)}\left[\underline{\Lambda}_{+}\left(x_{0}^{*}\right)-\frac{1}{2} \sigma^{2}(s) k_{-}^{\prime}(s)-b(s) k_{-}(s)-h(s)\right] m_{x_{0}^{*}}(d s)-k_{-}\left(x_{0}^{*}\right) \\
& =\int_{x_{0}^{*}}^{\bar{\chi}_{+}\left(x_{0}^{*}\right)}\left[\bar{\Lambda}_{+}\left(x_{0}^{*}\right)-\frac{1}{2} \sigma^{2}(s) k_{-}^{\prime}(s)-b(s) k_{-}(s)-h(s)\right] m_{x_{0}^{*}}(d s)-k_{-}\left(x_{0}^{*}\right) .
\end{aligned}
$$


These identities imply

$$
\begin{aligned}
0=\left[\bar{\Lambda}_{+}\left(x_{0}^{*}\right)-\underline{\Lambda}_{+}\left(x_{0}^{*}\right)\right] m_{x_{0}^{*}}\left(\left(x_{0}^{*}, \underline{\chi}_{+}\left(x_{0}^{*}\right)\right)\right) \\
\quad+\int_{\underline{\chi}_{+}}^{\bar{\chi}_{+}\left(x_{0}^{*}\right)}\left[\bar{\Lambda}_{+}\left(x_{0}^{*}\right)-\frac{1}{2} \sigma^{2}(s) k_{-}^{\prime}(s)-b(s) k_{-}(s)-h(s)\right] m_{x_{0}^{*}}(d s) .
\end{aligned}
$$

Also, combining (A.25) with Assumption 2.2(f), we can see that

$$
\bar{\Lambda}_{+}\left(x_{0}^{*}\right)-\frac{1}{2} \sigma^{2}(x) k_{-}^{\prime}(x)-b(x) k_{-}(x)-h(x)>0, \quad \forall x \in\left[a_{+}, \bar{\chi}_{+}\left(x_{0}^{*}\right)[.\right.
$$

However, this inequality, (A.33), and the fact that $\chi_{+}\left(x_{0}^{*}\right)>a_{+}$imply that the right-hand side of (A.35) is strictly positive, which is a contradiction.

The analysis above establishes that the functions $\Lambda_{+}$and $\chi_{+}$both are continuous and strictly increasing in $] A_{-}, \infty\left[\right.$. Also, this conclusion and the definition (A.30) of $A_{-}$imply that

$$
\left.\left.h\left(x_{0}\right)<\Lambda_{+}\left(x_{0}\right)<\Lambda_{+}(0), \quad \forall x_{0} \in\right] A_{-}, 0\right],
$$

which, combined with the fact that $\lim _{x \rightarrow-\infty} h(x)=\infty$ (see Assumption 2.2(b)), implies that $A_{-}>-\infty$.

We can prove all of the claims regarding the function $\chi_{-}$using similar reasoning. In particular, we can show that $\Lambda_{-}$and $\chi_{-}$both are continuous and strictly decreasing in the interval $]-\infty, A_{+}[$, where

$$
\left.A_{+}:=\sup \left\{x_{0} \in \mathbb{R} \mid \Lambda_{-}\left(x_{0}\right)>h\left(x_{0}\right)\right\} \in\right] 0, \infty[.
$$

Finally, the definitions of $A_{-}$and $A_{+}$in (A.30) and (A.38), respectively, the fact that $A_{+}$ and $A_{-}$both are real numbers, and the continuity of the functions $\Lambda_{+}, \Lambda_{-}$, and $h$ imply that

$$
\begin{aligned}
& \Lambda_{+}\left(A_{+}\right)>h\left(A_{+}\right)=\Lambda_{-}\left(A_{+}\right), \\
& \Lambda_{+}\left(A_{-}\right)=h\left(A_{-}\right)<\Lambda_{-}\left(A_{-}\right) .
\end{aligned}
$$

However, these inequalities and the fact that the function $\Lambda_{+}-\Lambda_{-}$is continuous and strictly increasing imply that there exists a unique $\left.x_{0} \in\right] A_{-}, A_{+}[$such that (A.17) holds true.

Proof of Lemma 3.1. Let $\tilde{x}_{0}$ and $\tilde{\lambda}$ be as in the statement of Lemma A.1. Since $\tilde{\lambda}>h\left(\tilde{x}_{0}\right)$, Assumption 2.2(b) implies that there exists exactly one point $x>\tilde{x}_{0}$ such that $h(x)=\tilde{\lambda}$. This fact, the observation that

$$
\frac{\partial g}{\partial x}\left(x, \tilde{\lambda}, \tilde{x}_{0}\right)=-\frac{2}{\sigma^{2}(x)}[h(x)-\tilde{\lambda}], \quad \forall x>\tilde{x}_{0} \text { such that } g\left(x, \tilde{\lambda}, \tilde{x}_{0}\right)=0
$$


the boundary condition $g\left(\tilde{x}_{0}, \tilde{\lambda}, \tilde{x}_{0}\right)=0$, and Assumption 2.2(b) imply that the equation $g\left(x, \tilde{\lambda}, \tilde{x}_{0}\right)=0$ can have at most one solution $\left.\hat{x}\left(\tilde{\lambda}, \tilde{x}_{0}\right) \in\right] \tilde{x}_{0}, \infty[$, and, if this solution exists, then either

$$
g\left(x, \tilde{\lambda}, \tilde{x}_{0}\right) \begin{cases}\geq 0, & \text { for } x \in\left[\tilde{x}_{0}, \hat{x}\left(\tilde{\lambda}, \tilde{x}_{0}\right)\right] \\ <0, & \text { for } x>\hat{x}\left(\tilde{\lambda}, \tilde{x}_{0}\right)\end{cases}
$$

or $g\left(x, \tilde{\lambda}, \tilde{x}_{0}\right) \geq 0$, for all $x \geq \tilde{x}_{0}$. However, these inequalities, (A.14), and the strict positivity of $k_{-}$imply that $\chi_{+}\left(\tilde{x}_{0}\right)<\hat{x}\left(\tilde{\lambda}, \tilde{x}_{0}\right)$ and

$$
g\left(x, \tilde{\lambda}, \tilde{x}_{0}\right) \in\left[0, k_{-}(x)\right], \quad \forall x \in\left[\tilde{x}_{0}, \chi_{+}\left(\tilde{x}_{0}\right)\right]
$$

Similarly, we can show that

$$
g\left(x, \tilde{\lambda}, \tilde{x}_{0}\right) \in\left[-k_{+}(x), 0\right], \quad \forall x \in\left[\chi_{-}\left(\tilde{x}_{0}\right), \tilde{x}_{0}\right]
$$

Now, let $w$ be the unique, modulo an additive constant, function defined by

$$
w^{\prime}(x):= \begin{cases}-k_{+}(x), & \text { for } x<\chi_{-}\left(\tilde{x}_{0}\right), \\ g\left(x, \tilde{\lambda}, \tilde{x}_{0}\right), & \text { for } x \in\left[\chi_{-}\left(\tilde{x}_{0}\right), \chi_{+}\left(\tilde{x}_{0}\right)\right] \\ k_{-}(x), & \text { for } x>\chi_{+}\left(\tilde{x}_{0}\right) .\end{cases}
$$

With regard to its construction, $w$ is $C^{2}$, is decreasing in ] $-\infty, \tilde{x}_{0}[$, and is increasing in ]$\tilde{x}_{0}, \infty[$. Furthermore, $w$ satisfies the HJB (3.1) provided

$$
\begin{gathered}
\frac{1}{2} \sigma^{2}(x) w^{\prime \prime}(x)+b(x) w^{\prime}(x)+h(x)-\lambda \geq 0, \quad \forall x<\chi_{-}\left(\tilde{x}_{0}\right), \\
\frac{1}{2} \sigma^{2}(x) w^{\prime \prime}(x)+b(x) w^{\prime}(x)+h(x)-\lambda \geq 0, \quad \forall x>\chi_{+}\left(\tilde{x}_{0}\right), \\
-k_{+}(x) \leq w^{\prime}(x) \leq k_{-}(x), \quad \forall x \in\left[\chi_{-}\left(\tilde{x}_{0}\right), \chi_{+}\left(\tilde{x}_{0}\right)\right], \\
w^{\prime}(x) \leq k_{-}(x), \quad \forall x<\chi_{-}\left(\tilde{x}_{0}\right), \\
w^{\prime}(x) \geq-k_{+}(x), \quad \forall x>\chi_{+}\left(\tilde{x}_{0}\right),
\end{gathered}
$$

are all true. In view of (A.42), (A.43), and the definition of $w$ in (A.44), inequality (A.47) is plainly true, while (A.48) and (A.49) follow trivially from the fact that $k_{+}$and $k_{-}$both are positive functions. Finally, we can easily see that (A.45) and (A.46) are true once we combine Assumptions 2.2(e, f) with (A.16) in Lemma A.1. 


\section{Acknowledgment}

This research was supported by EPSRC Grant no. GR/S22998/01. We are grateful to the anonymous referee and the editor for their comments that led to a significant improvement of the paper.

\section{References}

[1] J. Bather and H. Chernoff, Sequential decisions in the control of a spaceship, Proceedings of Fifth Berkeley Symposium on Mathematical Statistics and Probability (Berkeley, Calif., 1965/1966), Vol. III: Physical Sciences, University of California Press, California, 1967, pp. 181-207.

[2] V. E. Beneš, L. A. Shepp, and H. S. Witsenhausen, Some solvable stochastic control problems, Stochastics and Stochastics Reports 4 (1980), no. 1, 39-83.

[3] P. L. Chow, J.-L. Menaldi, and M. Robin, Additive control of stochastic linear systems with finite horizon, SIAM Journal on Control and Optimization 23 (1985), no. 6, 858-899.

[4] P. Dai Pra, G. B. Di Masi, and B. Trivellato, Pathwise optimality in stochastic control, SIAM Journal of Control and Optimization 39 (2000), no. 5, 1540-1557.

[5] P. Dai Pra, W. J. Runggaldier, and M. Tolotti, Pathwise optimality for benchmark tracking, IEEE Transactions on Automatic Control 49 (2004), no. 3, 386-395.

[6] M. H. A. Davis and M. Zervos, A pair of explicitly solvable singular stochastic control problems, Applied Mathematics and Optimization 38 (1998), no. 3, 327-352.

[7] W. H. Fleming and H. M. Soner, Controlled Markov Processes and Viscosity Solutions, Applications of Mathematics (New York), vol. 25, Springer, New York, 1993.

[8] J. M. Harrison and M. I. Taksar, Instantaneous control of Brownian motion, Mathematics of Operations Research 8 (1983), no. 3, 439-453.

[9] I. Karatzas, A class of singular stochastic control problems, Advances in Applied Probability 15 (1983), no. 2, 225-254.

[10] I. Karatzas and S. E. Shreve, Brownian Motion and Stochastic Calculus, Graduate Texts in Mathematics, vol. 113, Springer, New York, 1988.

[11] J. Ma, On the principle of smooth fit for a class of singular stochastic control problems for diffusions, SIAM Journal on Control and Optimization 30 (1992), no. 4, 975-999.

[12] J.-L. Menaldi and M. Robin, Some singular control problem with long term average criterion, System Modelling and Optimization (Copenhagen, 1983), Lecture Notes in Control and Information Sciences, vol. 59, Springer, Berlin, 1984, pp. 424-432.

[13] È. Presman, V. I. Rotar, and M. Taksar, Optimality in probability and almost surely. The general scheme and a linear regulator problem, Stochastics and Stochastics Reports 43 (1993), no. 3-4, $127-137$.

[14] D. Revuz and M. Yor, Continuous Martingales and Brownian Motion, 2nd ed., Fundamental Principles of Mathematical Sciences, vol. 293, Springer, Berlin, 1994.

[15] V. I. Rotar, Connectivity property and optimality almost surely and in probability, New Trends in Probability and Statistics, Vol. 1 (Bakuriani, 1990), VSP, Utrecht, 1991, pp. 528-539.

[16] W. Schmidt, On stochastic differential equations with reflecting barriers, Mathematische Nachrichten 142 (1989), 135-148.

[17] S. E. Shreve, J. P. Lehoczky, and D. P. Gaver, Optimal consumption for general diffusions with absorbing and reflecting barriers, SIAM Journal on Control and Optimization 22 (1984), no. 1, $55-75$.

[18] H. M. Soner and S. E. Shreve, Regularity of the value function for a two-dimensional singular stochastic control problem, SIAM Journal on Control and Optimization 27 (1989), no. 4, 876907. 
[19] M. Sun, Singular control problems in bounded intervals, Stochastics and Stochastics Reports 21 (1987), no. 4, 303-344.

[20] A. P. N. Weerasinghe, Stationary stochastic control for Itô processes, Advances in Applied Probability 34 (2002), no. 1, 128-140.

[21] H. Zhu, Generalized solution in singular stochastic control: the nondegenerate problem, Applied Mathematics and Optimization 25 (1992), no. 3, 225-245.

Andrew Jack: Department of Mathematics, King's College London, Strand, London WC2R 2LS, UK E-mail address: andrew.j.jack@kcl.ac.uk

Mihail Zervos: Department of Mathematics, King's College London, Strand, London WC2R 2LS, UK E-mail address: mihail.zervos@kcl.ac.uk 


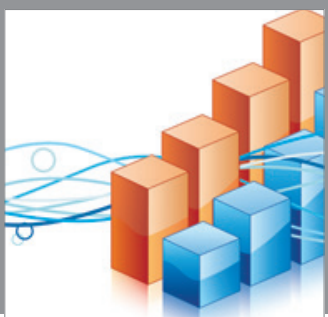

Advances in

Operations Research

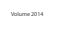

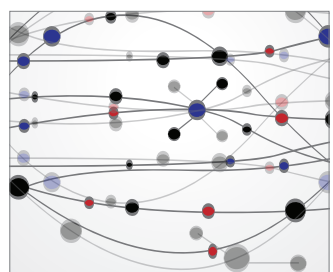

\section{The Scientific} World Journal
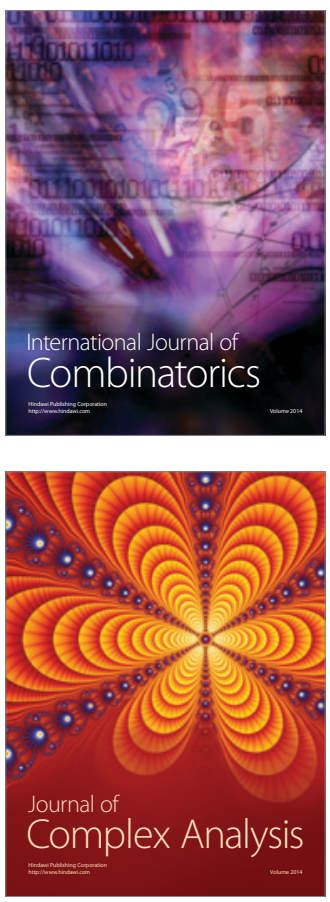

International Journal of

Mathematics and

Mathematical

Sciences
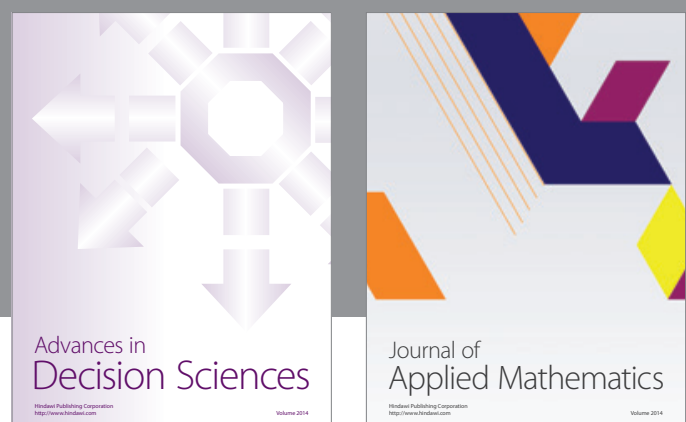

Journal of

Applied Mathematics
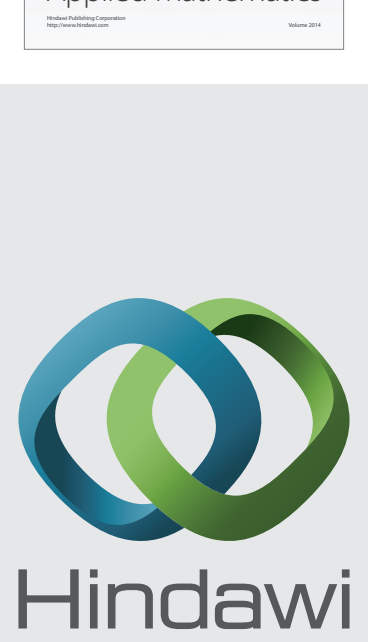

Submit your manuscripts at http://www.hindawi.com
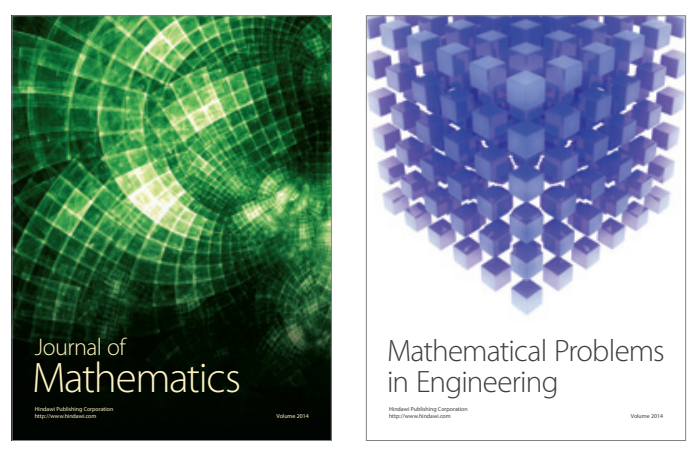

Mathematical Problems in Engineering
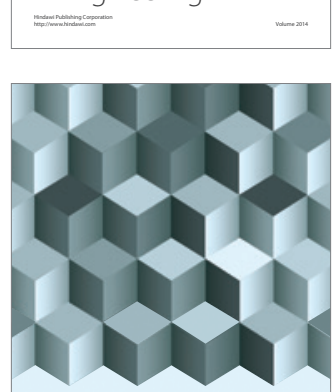

Journal of

Function Spaces
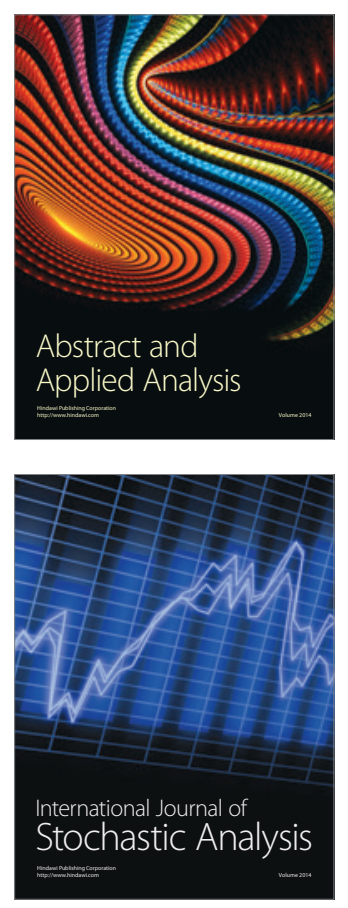

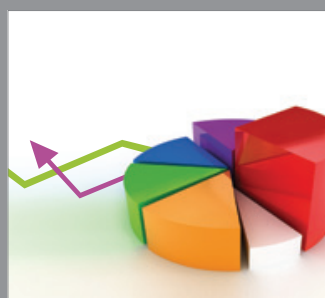

ournal of

Probability and Statistics

Promensencen
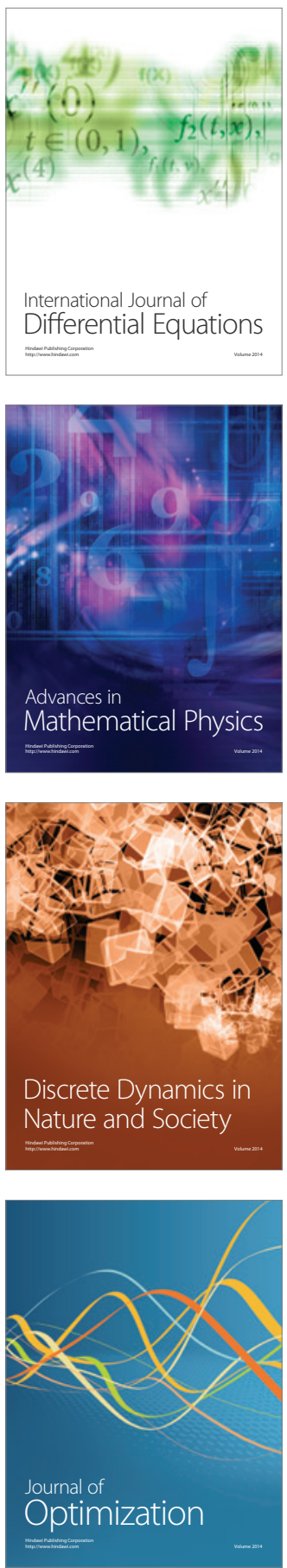